\title{
Late Onset Pantothenate Kinase-Associated Neurodegeneration: Case Report
}

ISSN: 2637-7748

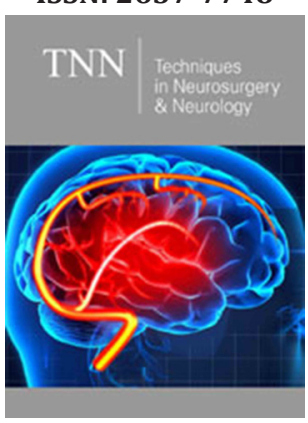

*Corresponding author: Luis Torres Ramírez, Instituto Nacional de Ciencias Neurológicas- Department of Neurodegenerative Diseases, Lima - Perú

\section{Submission: 酮 October 22, 2020}

Published: 此 November 13, 2020

Volume 3 - Issue 4

How to cite this article: Torres $L^{*}$, Quispe D, Flores M, Vélez M and Cosentino C. Late Onset Pantothenate Kinase-Associated Neurodegeneration: Case Report. Tech Neurosurg Neurol. 3(4). TNN. 000567. 2020.

DOI: $10.31031 /$ TNN.2020.03.000567

Copyright@ Luis Torres Ramírez, Gonzalez EKB and Esqueda LMA, This article is distributed under the terms of the Creative Commons Attribution 4.0 International License, which permits unrestricted use and redistribution provided that the original author and source are credited.

\section{Torres L*, Quispe D, Flores M, Vélez M and Cosentino C}

Instituto Nacional de Ciencias Neurológicas- Department of Neurodegenerative Diseases, Lima - Perú

\begin{abstract}
Introduction: Pantothenate kinase-associated neurodegeneration (PKAN) is a neurodegenerative disease due to abnormal basal ganglia iron accumulation, has a type of autosomal recessive transmission and is caused by mutations in the gene encoding pantothenate kinase 2 enzyme (PANK2) which is located on chromosome.
\end{abstract}

Case report: A 30-year-old-male patient is presented, without family history with similar disease. The predominant clinical manifestation was late, sporadic and progressive appearance of a persistent generalized dystonia associated with Parkinsonism.Brain MRI in axial T2 and FLAIR sequences showed marked hypo intensity in both globus pallidus with hyperintensity in their centers ("eye of tiger" sign). Genetic analysis was consistent with the clinical suspicion of PKAN by heterozygous mutation of the gene encoding the PKAN2: Exon 5: c.1453insC p. Ser485GlnfsX1 and Exon 7: c.1709C/T p.Prol570Leu (rs41279408).

Conclusion: We present the first case in our country of late-onset PKAN with molecular diagnosis.

Keywords: Dystonia; PKAN2; Eye of tiger

\section{Introduction}

Pantothenate kinase-associated neurodegeneration (PKAN) is one of the ten clinical forms of neurodegeneration with brain iron accumulation, representing the $50 \%$ of all the cases, characterized by progressive neurological symptoms related with abnormal basal ganglia iron accumulation [1,2]. PKAN is an autosomal recessive disorder caused by mutation of the pantothenate kinase 2 gene localized in chromosome 20p12.3-p13 [3,4]. The prevalence is estimated in 1-3 cases per million, differentiating between them in early onset PKAN, fast progressive "classic" disease, and atypical form or late onset PKAN with slow progression [5]. Diagnostic criteria of PKAN were described by Dooling et al. [6]. based in its clinical characteristics and anatomopathological findings [6]. 75\% of all of PKAN cases begin in the childhood, usually at 6 years old ( 6 months to 12 years age range), the remainders are atypical forms of presentation with an average of 14 years (1 to 28 years age range) [7]. In LatinAmerican, atypical or late onset PKAN has been reported in Brazil with 5 cases, all of them with the typical images of "eye of tiger" sign in the brain MRI [8-10]. We present the first late onset PKAN case with molecular diagnosis in our country.

\section{Case Report}

A 30-year-old-patient, without family history with similar disease, with a regular child development, unfinished high school, cocaine, and marijuana consume only three times twelve years ago. He realized behavioral changes, irritability, and aggressiveness with his relatives in the last two years until now. Sixteen months before his admission presents action tremor in left foot, six months after in right foot too, action tremor first, then rest tremor, involving all the right lower extremity. Left lower extremity tremor disappeared spontaneously. Eight months ago, painful retrocollis appears with right rotation and back hyperextension, balancing the movement with strange axis position six months ago. Biperiden $4 \mathrm{mg} /$ day improved right lower extremity and cervical dystonia. Biperiden $6 \mathrm{mg} /$ day was not better. Neurological examination: Alert, fluent and clear conversation, collaborative. Retrocollis involves neck and trunk in rest, dystonic right foot, and rest tremor in lower extremities, that worsening in posture, walking and stress (Figure 1). Active movements are preserved, normoreflexia, Babinski's sign absent. No cerebellar, sensitivity or cranial 
nerves involvement. Ancillary tests: Peripheral blood smear study looking for acanthocytes was normal, ceruloplasmin, 24-hour urine cooper and ferritin tests were normal. Electromyography-nerve conduction velocity studies showed frequent spontaneous negative activity in neck muscles (scalene, splenius capitis, upper trapezius, and sternocleidomastoid muscles), corresponding with dystonia in these groups of muscles. Neuro-ophthalmic examination dismissed retinitis pigmentosa and Kayser-Fleischer ring. Mini-mental status exam score was 21/30 points. Neuropsychological examination showed mental skills affected with a "very low" mental function. T2- weighted and FLAIR MRI sequences showed noticeable low signal both pallidus nucleus with high center ("eyes of tiger" sign) (Figure 2). Genetical test confirmed the clinical suspect of PKAN because a heterocygotic PKAN2 gene mutation: Exon 5: c.1453insC p. Ser485GlnfsX1 and exon 7: c.1709C/T p. Pro570Leu (rs41279408) ((Figure 3). Treatment: Biperiden 4mg/day, clonazepam 12mg/day, well tolerated but with mild clinical response. Because dystonia got worsening even though the treatment, we decided use botulinum toxin type A 100UI applying it in back neck region muscles, with $60 \%$ daily activities improvement seven days after injection.



Figure 1: It is clear severe paravertebral muscles dystonia conditioning hyperextension of the neck and trunk.

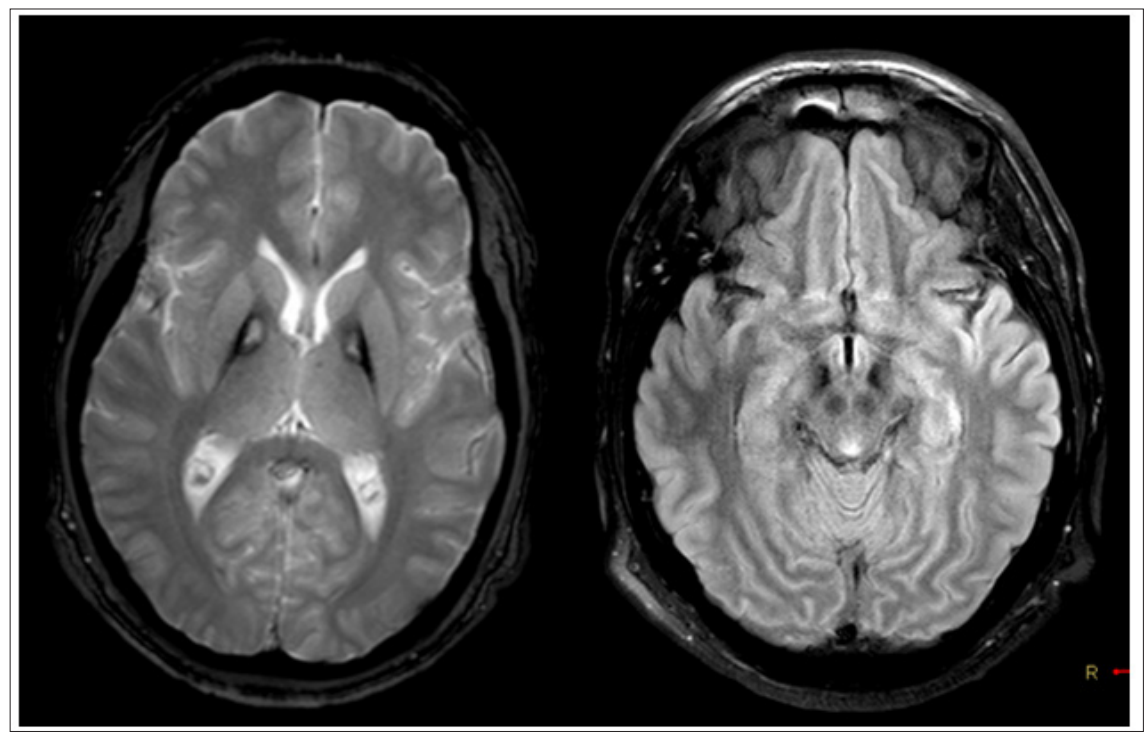

Figure 2: Left: T2-MRI image shows bilateral pallidum hypo intensity with a central hyperintensity area, corresponding with "eye of tiger" sign. Right: FLAIR-MRI image shows hypo intensity in substantial nigra. 

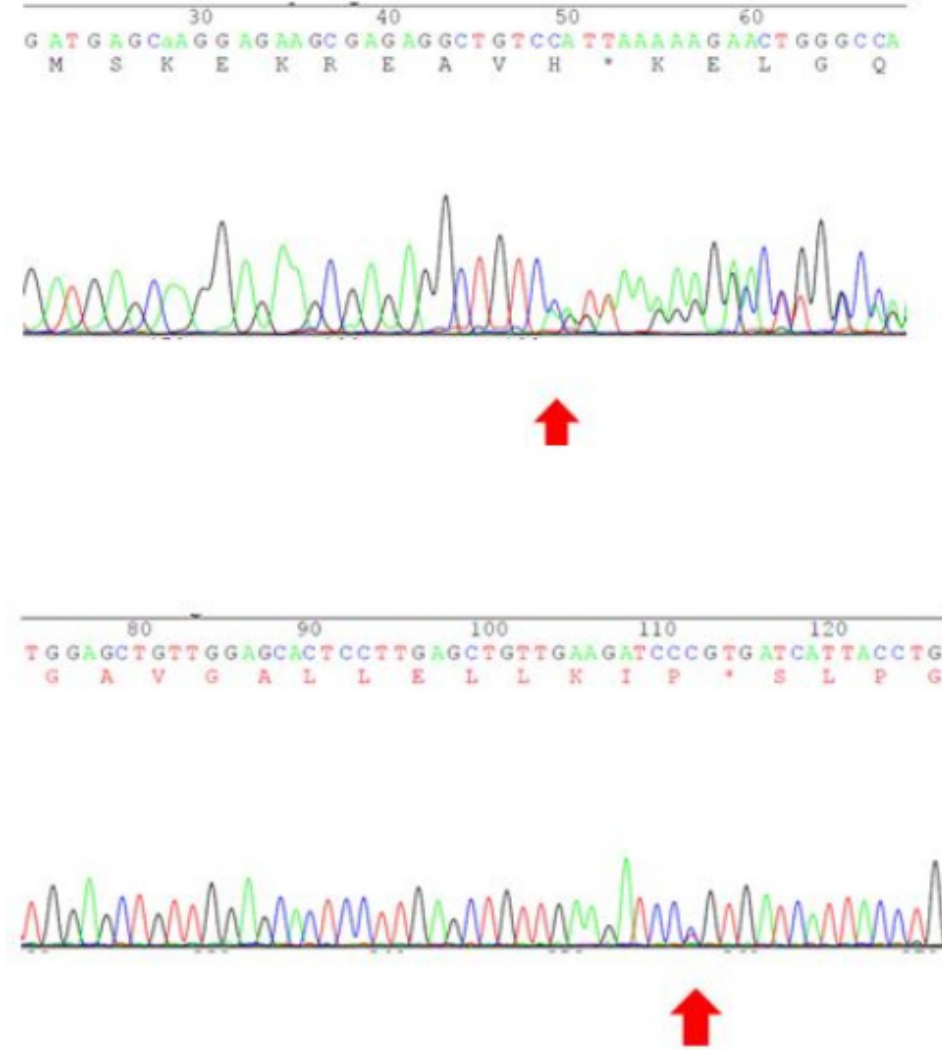

Figure 3: Upper: Exon 5: c1453insC p. Ser485GlnfsX1. Lower: Exon 7: c.1709C/T p. Pro570Leu (rs41279408).

\section{Discussion}

PKAN, previously named Hallervorden-Spatz syndrome, is one of the ten neurodegenerations with brain iron accumulation subtypes, many of which have an inherited recessive pattern affecting child mainly [1]. Neuroferritinopathy and aceruloplasminemy affect an adult, but ferritin and ceruloplasmin levels are normal in our patient. Other more common movement disorders which could affect young people are the neurocanthocytosis and Wilson's disease. In our patient, the peripheral blood smear study didn't show acanthocyte, and 24-hour cooper blood level was normal. In a 52-patient case series report with late onset of the disease, dystonia was the most common symptom (87\%), affecting cranial muscles and upper and lower extremities, axial dystonia, dysarthria, rigidity and choreoathetosis later. Spasticity, hyperreflexia and plantar extensor response were present in $25 \%$ of the patients and cognitive impairment in 29\% [5]. The most important clinical symptom of our patient was the onset of persistent sporadic generalized dystonia related with Parkinsonism (dystonia-parkinsonism syndrome) [11], correlated with Hayflick case series findings [5]. Neuropsychiatric signs were present from the beginning like most of the late onset cases. Retinitis pigmentosa could be present with less frequency in this kind of patients. Walking impairment appears 15 to 40 years after the beginning of symptoms [12]. In our case its presence during ophthalmological examination was dismissed.

Wide hypointense pallidal signal corresponding with iron deposit is the brain MRI characteristic finding, with T2 and FLAIR central hyperintense focus (gliosis zone). Our patient images showed substance nigra hypointense signal in T2 and FLAIR corresponding with iron deposit. In a case series studied by Hayflick [5] showed the "eyes of tiger" sign is strongly correlated with PANK2 gene mutation. This mutation was founded in more than third of patients with late onset PKAN (5) [13]. Additionally, patients who don't express this mutation, only show hypo signal in both globus pallidum in $\mathrm{T} 2$, cerebellar atrophy and iron deposit red nucleus and dentate nucleus [5]. PANK2 gene codifies enzyme panthotenato kinasa 2 and its variation causes defect in pantothenic acid phosphorylation (process required to coenzyme A synthesis), essential intermediary in the fatty acid metabolism. Cysteine storing in globus pallidum, resulting in phosphopantothenate reduction, causes iron chelation and oxidative damage (basal ganglia nuclei impairment is related with pantothenate kinase receptors in excess). The discovery of two mutations in two different alleles of PKAN2 genes in unusual, so we assume that it could be a new mutation we haven't found in another articles. To be confirmed, we have to study and identify our patient parents' mutation and doing a sequence of the complementary bases chain of DNA. Treatment is symptomatic, deserving to be mentioned botullinum toxin, oral or intrathecal baclofen, ablative pallidotomy, deep brain stimulation and physical therapy [1,7]. A previous study with deferiprone showed decrease of brain iron concentration, but without modify the clinical course of the disease [14]. In our patient, the symptomatic treatment was biperiden and clonazepam, plus botullinum toxin A, improving cervical dystonia. In our country, Torres et al. [15] published four probable cases of 
NAPK in children, two of them with consanguinity history [15], being our case the first to be notified like late onset NAPK with molecular diagnostic.

\section{Acknowledgement}

We want to express our gratitude for Neurogenetic Unit of Instituto Nacional de Ciencias Neurológicas, Lima, Perú, specially Dr. Mario Cornejo because his invaluable support, and for Dr. Tu-Hsueh Yeh, Chief of the Division of Movement Disorders, Department of Neurology, Chang Gung Memorial Hospital, Taipei, Taiwan, who generously made the molecular diagnosis.

\section{References}

1. Hogarth P (2015) Neurodegeneration with brain Iron accumulation: diagnosis and management. J Mov Disord 8(1): 1-13.

2. Kurian MA, Hayflick SJ (2013) Pantothenate kinase-associated neurodegeneration (PKAN) and PLA2G6-associated neurodegeneration (PLAN): review of two major neurodegeneration with brain iron accumulation (NBIA) phenotypes. Int Rev Neurobiol 110: 49-71.

3. Taylor TD, Litt M, Kramer P, Pandolfo M, Angelini L, et al. (1996) Homozygosity mapping of Hallervorden-Spatz syndrome to chromosome. Nat Genet 14(4): 479-481.

4. Zhou B, Westaway SK, Levinson B, Johnson MA, Gitschier J, et al. (2001) A novel pantothenate kinase gene (PANK2) is defective in HallervordenSpatz syndrome. Nat Genet 28(4): 345-349.

5. Hayflick SJ, Westaway SK, Levinson B, Zhou B, Johnson MA, et al. (2003) Genetic, clinical, and radiographic delineation of Hallervorden Spatz Syndrome. N Engl J Med 348(1): 33-40.
6. Dooling EC, Schoene WC, Richardson EP (1974) Hallervorden-Spatz syndrome. Arch Neurol 30(1): 70-83.

7. Gregory A, Hayflick SJ (1993-2019) Pantothenate kinase-associated neurodegeneration. In: Adam MP, Ardinger HH, Pagon RA, et al. (Eds.), GeneReviews ${ }^{\circledR}$, USA.

8. Carod AFJ, Vargas AP, Marinho PB, Fernandes STV (2004) Tourettism, hemiballism and juvenile Parkinsonism: expanding the clinical spectrum of the neurodegeneration associated to pantothenate kinase deficiency (Hallervorden Spatz syndrome). Rev Neurol 38(4): 327-331.

9. Barbosa ER, Bittar MS, Bacheschi LA, Comerlatti LR, Scaff M (1995) Precocious Parkinson's disease associated with eye-of-the-tiger type pallidal lesions. Arq Neuropsiquiatr 53(2): 294-297.

10. Adamo JJ (1993) Hallervorden Spatz syndrome: report of a case. Arq Neuropsiquiatr 51(1): 130-133.

11. Albanese A, Bhatia K, Bressman SB, Delong MR, Fahn S, et al. (2013) Phenomenology and classification of dystonia: a consensus update. Mov Disord 28(7): 863-873.

12. Gregory A, Polster BJ, Hayflick SJ (2009) Clinical and genetic delineation of neurodegeneration with brain iron accumulation. J Med Genet 46(2): 73-80.

13. Popławska DK, Florczak WJ, Kozubski W (2014) Update on neurodegeneration with brain iron accumulation. Neurol Neurochir Pol 48(3): 206-213.

14. Abbruzzese G, Cossu G, Balocco M, Marchese R, Murgia D, et al. (2011) A pilot trial of deferiprone for neurodegeneration with brain iron accumulation. Hematologica 96(11): 1708-1711.

15. Torres L,Cosentino C, Mori N, Vélez M (2012) Probableneurodegeneración asociada a pantotenato kinasa (PKAN) Estudio de cuatro casos en Perú. Diagnóstico 51(1): 46-51. 